\title{
Approaches to monitoring natural and anthropogenic objects in an analysis of the environment around large industrial facilities
}

\author{
Anna M. Konstantinova ${ }^{1}$, Evgeny A. Loupian ${ }^{1}$, Ivan V. Balashov ${ }^{1}$ and \\ Alexandr V. Kashnitskii ${ }^{1}$ \\ ${ }^{1}$ Space Research Institute of the Russian Academy of Sciences (IKI RAS), Moscow, Russia
}

\begin{abstract}
The paper deals with the problems of a sharp increase in the number of remote sensing satellite systems and the amount of data received, and because of this, the need to develop new approaches to the processing and use of satellite information. An "object-oriented" approach to work with remote sensing data for monitoring natural and anthropogenic processes is proposed. The paper presents a subsystem for working with objects of observation created on the basis of the "IKI-Monitoring" Center for Collective Use for the implementation of this approach. The efficiency of the subsystem is confirmed by examples of its use for the organization of remote sensing of zones of potential contamination from large industrial facilities.
\end{abstract}

\section{Keywords}

Remote sensing, data processing, satellite data, environment, man-made dumps, industrial facilities, spectral index, "IKI-Monitoring" Center for Collective Use.

\section{Introduction}

In the modern world, satellite monitoring systems are actively used to solve various scientific and applied problems, to study natural resources and anthropogenic processes and objects. Every day more and more opportunities are opening up for environmental research due to the constant growth of the number remote sensing satellite systems and the improvement the quality of the data they provide. In the future, a significant increase of the number of remote sensing satellites is planned due to the commissioning of observation systems built on small spacecraft. Such trends lead to a sharp increase in the amount of information received, and require the development of fundamentally new approaches for archiving, storing and working with it.

One of these new approaches can be an "object-oriented" approach to the use of remote sensing data. The basis of the described approach is working with objects of observation, which allows to reduce the amount of information stored for analysis and to easily identify changes occurring with objects, in contrast to the traditional pixel-by-pixel analysis of the territory. The key difference is that the data is not stored for each pixel separately, but as averaged values

SDM-2021: All-Russian conference, August 24-27, 2021, Novosibirsk, Russia

$\bigoplus$ konstantinova.anouk@gmail.com (A. M. Konstantinova)

(c) (1) ๑ 2021 Copyright for this paper by its authors. Use permitted under Creative Commons License Attribution 4.0 International (CC BY 4.0).

CEUR Workshop Proceedings (CEUR-WS.org) 
for the entire contour of the object under study or other homogeneous area. This approach is aimed at creating an almost continuous long-term data series for the objects under study.

For this purpose, the IKI RAS developed an automated tool for monitoring the state of natural and natural-anthropogenic objects ObjectsSurveysSMIS [1], operating on the basis of the «IKIMonitoring» Center for Collective Use [2]. It was integrated into the information services VEGA-Constellation (http://sozvezdie-vega.ru), developed at the IKI RAS. Retrospective and operational monitoring of objects of observation is carried out in automatic mode by using this tool. Based on long-term series of satellite data of different spatial resolution, the indicators selected for control (spectral indices, values in channels) are calculated for the polygons of objects for further analysis of the time series of their values.

The article provides examples of the applicability of this approach and tool to identifying the impact of industrial waste zones and dumps on the environment. This impact is mainly manifested in changes in the vegetation cover in areas where large industrial facilities are located, as well as in the state of water bodies. Comparison of the values of indicators of potentially "polluted" and "control" (reference) sites of various types of territory within the same area of pollution made it possible to identify negative changes in the environment caused by the activities of large industrial enterprises.

\section{Subsystem requirements}

To implement the technology of "object-oriented" monitoring, the following tools were to be developed and the following archives were maintained:

- tools for establishing and describing new objects of observation that have a different nature of origin, indicating for them an unlimited number of attributes, calculated parameters, observation interval for sampling scenes of satellite data from archives;

- a module for automated calculation of various characteristics for polygons of objects on the basis of operational and historical satellite information available in the archives of the IKI Monitoring Center;

- automated maintenance of object-oriented databases (DB) containing information about objects of observation, their polygons, including those with dynamic boundaries, calculated characteristics, averaged over polygons of objects;

- tools for analyzing information on objects calculated on the basis of satellite data, including visualization of calculated values on a map and building time series, tools for joint analysis of objects;

- administration tools that allow you to easily describe and configure the rules for monitoring different types of objects based on different satellite information to ensure the same type of automated work both with objects of different nature and with multiple satellite systems.

\section{Subsystem structure}

Thus, to solve the problem of "object-oriented" monitoring on the basis of the above requirements, three main directions for development can be distinguished: service interfaces with 
which experts and administrators will work, means of maintaining a database of objects and an annotation module (automated calculation of characteristics).

In particular, among the service interfaces, the following should be implemented:

- an interface that allows you to work with objects of observation: create new ones, edit existing ones, go to the interface for analyzing characteristics;

- map interface for spatial visualization and analysis of various characteristics of objects;

- interface for analyzing time series of characteristics of various objects with the ability to automatically upload data to tabular interfaces;

- interface for setting new characteristics (indicators) and rules for calculating statistics based on satellite information.

In turn, the means of maintaining the database necessary to ensure the work of experts should consist of:

- database of objects of observation with the specified parameters and values of characteristics;

- database of spectral indices (indicators) with a description of the rules by which it is necessary to calculate them (satellite systems, information products, etc.), visualize their values on graphs and a map.

Consequently, the created subsystem of "object-oriented" monitoring should have the following form and consist of the following functional blocks, shown in Figure 1.

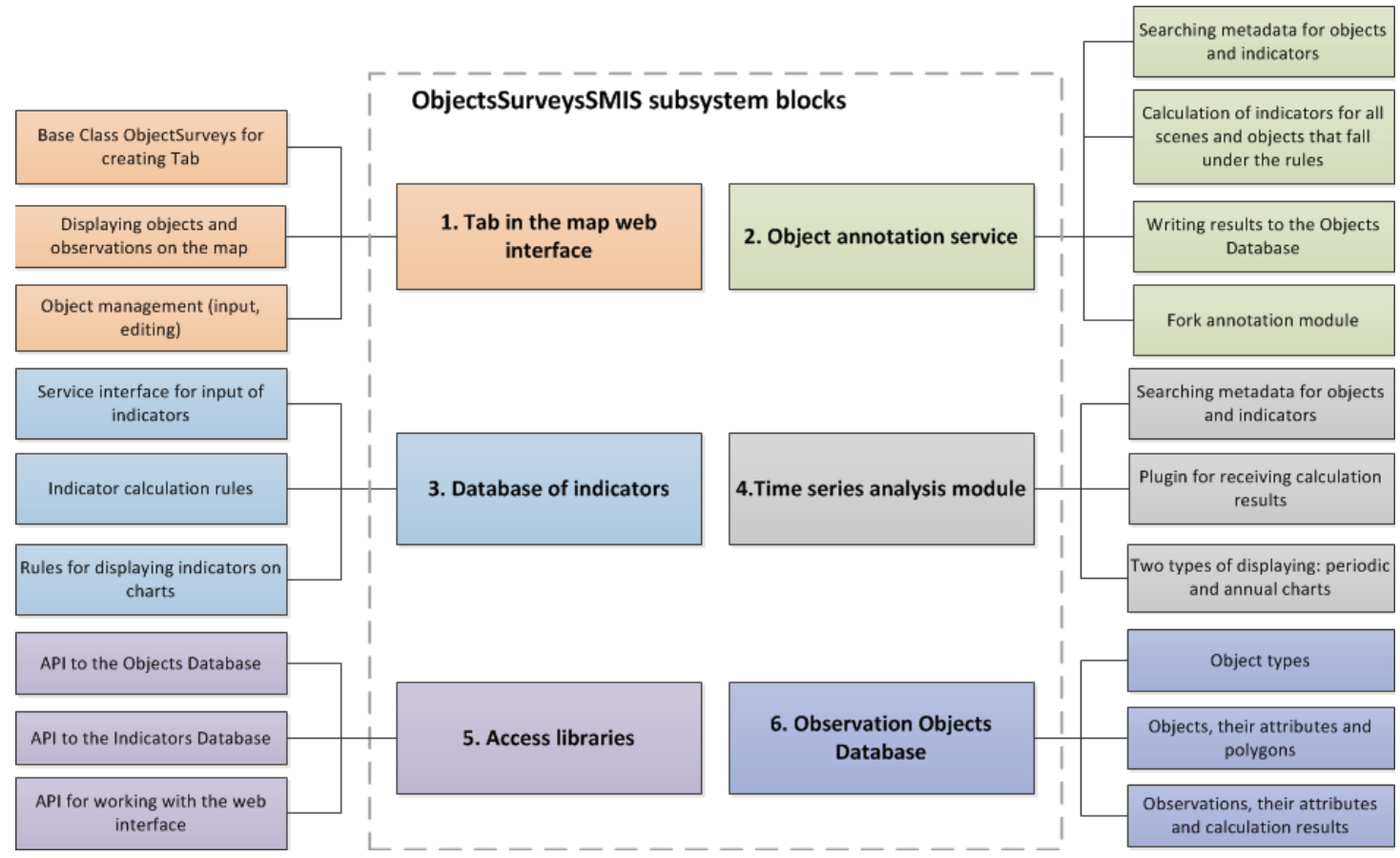

Figure 1: Subsystem structure. 
The described technology should have such an architecture and connections with external technologies so that it can be used to easily monitor objects of various types and solve research problems of a wide range. Therefore, it is necessary to be able at any time to create a new type of objects of observation and the rules for working with it, to indicate characteristics for it for calculation based on satellite data (choose from existing ones or create new ones), visualization parameters for calculated values, etc. The entered information must be assimilated in various blocks of the subsystem, and access to it must be provided from each block.

It was decided to take the ObjectsServeysSMIS_les software package (SP), developed at IKI RAS for remote monitoring of forest conditions, as the basis for maintaining the database of objects of observation.

To create the rest of the functional blocks of the subsystem, technologies developed at the IKI RAS were also involved. However, the above SP and other technologies involved did not provide the necessary functionality to create the subsystem. To support it, new functional blocks have been created and existing ones have been expanded. In particular, the development resulted in the creation of:

- unified automated service for calculating various characteristics of objects based on satellite data (annotation module).

- a unified scheme for describing indicators (based on spectral indices and information products available in map interfaces). Such a scheme should not only allow specifying the characteristics that must be calculated for objects, but also provide end-to-end integration of data on indicators in the cartographic interface, the graphical analysis tool, the annotation module;

- a specialized section in the map interface for working with objects and their observations;

- means of integrating the calculated values into the graphing subsystem for analyzing the time series of observations of objects;

- means of visualization of the values of the calculated indicators on the map.

Thus, on the basis of the ObjectsServeysSMIS_les SP, a subsystem for observing objects was created, called ObjectsSurveysSMIS, which provides a comprehensive solution to problems arising from monitoring a wide class of different types of objects. The subsystem was integrated into the Vega-Science information system (IS) http://sci-vega.ru (the Constellation-Vega family), and the data necessary for calculating the indicators are provided by services and archives implemented within the framework of the IKI-Monitoring Center for Collective Use.

\section{The main functionality of the monitoring subsystem}

To organize the direct monitoring of objects using satellite data, an automated annotation service was developed. It should be noted that it works in two modes: calculation based on historical scenes of satellite data for new objects and calculation based on operational data appearing in archives for already established objects. The process for searching for new objects is launched every minute, and the process for searching for new data is launched daily.

The input of the annotation process receives information about the type of annotated objects and the launch mode, then a search for objects that meet the specified conditions is performed. 
For the specified type of object, a list of calculated indicators from the database of indicators and information about satellite systems, the data of which can be used for calculation, described in the data sources of indicators, are requested, then query blocks are formed to search for satellite scenes in the distributed archives of the IKI-Monitoring Center for Collective Use that meet the specified conditions. For all satellite data scenes, cloud masks are loaded multithreaded if needed. Then, in a parallel stream, tiff files are loaded with the values of the indicator for all scenes, the tiff files are filtered by the cloud mask, the necessary characteristics are calculated, and the values are entered into the database of observation objects.

The collection of statistics in automatic mode inside the polygon of one new object on the scenes of satellite data available in distributed archives takes about a few minutes.

A unified scheme for describing indicators used for monitoring objects of observation was created on the basis of a specialized database of indicators with a flexible structure. This database allows you to maintain general information about all available indicators for each type of annotated objects, as well as to define an arbitrary set of necessary parameters for each of the indicators. To ensure the integration and assimilation of the rules for working with a new indicator in all functional blocks of the technology being created, it is necessary to define two display types for it in the service interface of the indicators database, describing the rules for calculating it in the annotation service and the rules for displaying the calculation results on graphs.

To automatically add the calculated data for a new indicator to the time series analysis interface, you need to define the following information for it in the rules for displaying the indicator on charts: axis description, data duty cycle, module for requesting data, data captions.

To establish a new object using this specialized tab in the map web interface, an expert needs only to outline the object on the map, indicate its type and range of observation. Further, the new object is picked up by the annotation service, and after some time of calculation, the expert has the opportunity to visualize the calculated values on the map by selecting an object in the list of objects and a scene of satellite data, or to analyze the received data in the time series analysis module, to unload the values into tabular interfaces.

\section{Examples}

To date, more than 20 districts of the location around large industrial facilities have been added to the Vega-Science IS. In each district, pollution sources (waste, dumps, etc.), as well as potentially "polluted" and "control" sites among vegetation and water bodies, are outlined. For each of the sites, using the subsystem ObjectsSurveysSMIS, control indicators were calculated for the entire observation period. This section presents the results of calculations obtained for some of the study districts [3]: in one of the examples the impact on vegetation is assessed, in the other on water bodies.

\subsection{Kachkanarsky GOK}

The Kachkanarsky GOK is given as the area in which the degradation of vegetation was assessed. The data of satellite remote monitoring of the dumps of the Kachkanarsky GOK on the environment were used to compare the long-term NDVI profiles obtained using MODIS device 
and calculated for homogeneous areas of birch and pine, located in and outside the polluted zones of the GOK. For this, homogeneous sites with a predominance of birch were selected: site 1 and site 2, located $500 \mathrm{~m}$ from the border of the dump of the Kachkanarsky GOK, site 3, located $3 \mathrm{~km}$ from the dump of this GOK, as well as site of pine 4, located between the quarry and the railway and car dumps.

Perennial profiles of birch plots are shown in Figure 2. It is important to note the practically unchanged NDVI values during the maximum vegetation period (July-August) for the control (reference) site, which is practically not affected by emissions from the Kachkanarsky GOK. At the same time, in areas exposed to pollution, a significant decrease in NDVI is observed. This is primarily seen in section 1 , located at a distance of $500 \mathrm{~m}$ from the dump boundary. Attention should also be paid to the fact that the degradation of the vegetation cover.

Perennial profiles of pine areas are shown in Figure 3. And here, there are practically unchanged NDVI values during the maximum vegetation period (July-August) for the control (reference) site, which is practically not affected by emissions from the Kachkanarsky GOK. At the same time, a significant decrease in NDVI is observed in section 4, which is subject to the influence of pollution. Here you should also pay attention to the fact that the degradation of the vegetation cover continues throughout the entire observation period, including at the present time.

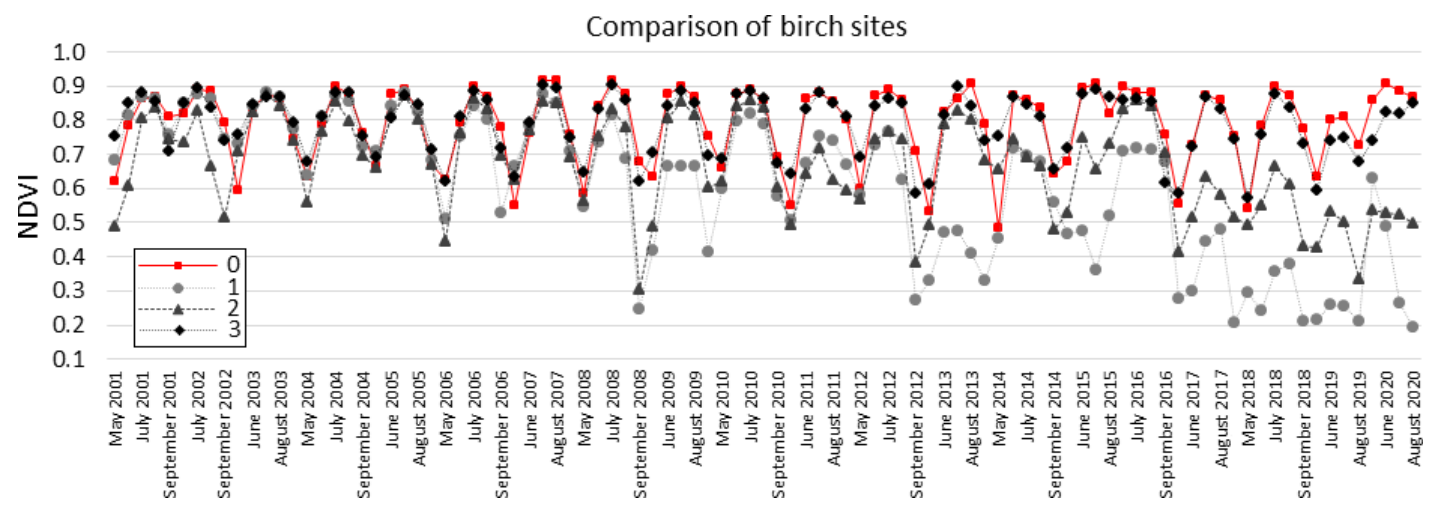

Figure 2: NDVI long-term rows of homogeneous birch areas: $0-$ control (reference) site; $1-$ site $1 ; 2-$ site $2 ; 3-$ site 3 .

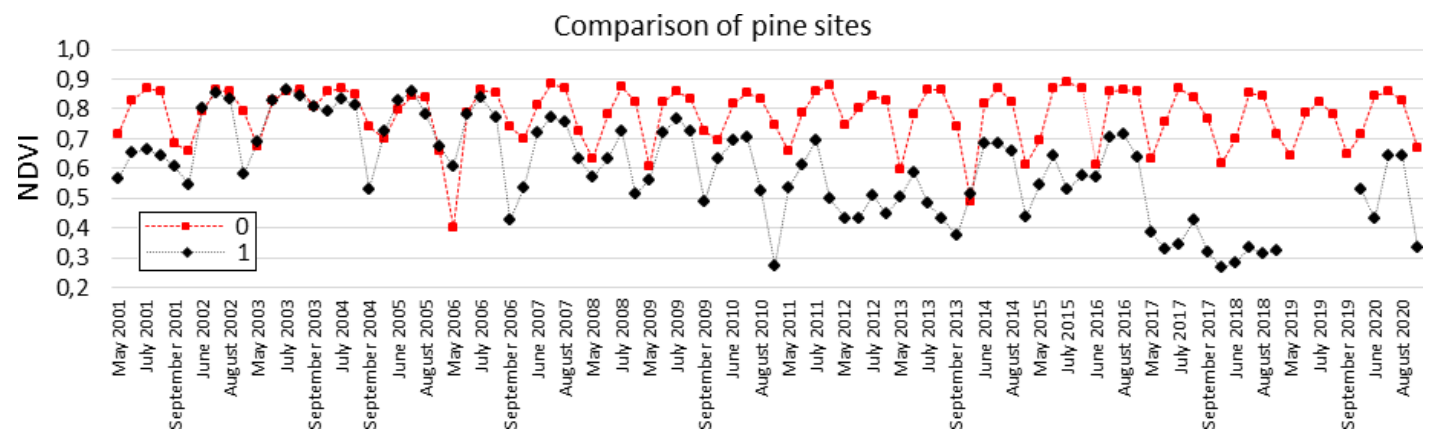

Figure 3: NDVI perennial rows of homogeneous pine areas: $0-$ control (reference) site; $1-$ site 4 . 


\subsection{Novoe tailing dump}

As an example of approaches to assessing the impact on water bodies, we consider the vicinity of the Novoye tailing dump, which is used for storing waste from the Talnakh enrichment plant "Norilsk Nickel". Attempts have been made to detect discharges or leaks from the tailings dam that pollute rivers and natural bodies of water. As mentioned above, using the created subsystem, it is possible to calculate a set of spectral indices that describe the state of water bodies, including the presence of pollutants in water bodies. AWEI1 (Automated Water Extraction Index) was experimentally chosen as one of such indicative indices. This index is calculated by the ratio

$$
\text { AWEI }=4 \times(\text { GREEN }- \text { SWIR1 })-(0.25 \times \mathrm{NIR}+2.75 \times \mathrm{SWIR} 2),
$$

where GREEN, NIR, SWIR1, SWIR2 are the reflection coefficients of the water surface in the corresponding spectral range. The peaks in the values of this index $(>0.3)$ correspond to the unnatural color (pollution) of the reservoir.

Figure 4 shows the data obtained using the AWEI1 index based on the channel data of the Sentinel-2 satellites, reflecting the percentage of polluted weeks for the snowless observation period from 2017 to 2020 for each studied reservoir. It should be noted that the Novoe tailing dump was built as part of the modernization of the Talnakh concentrator in 2015-2017, i.e.

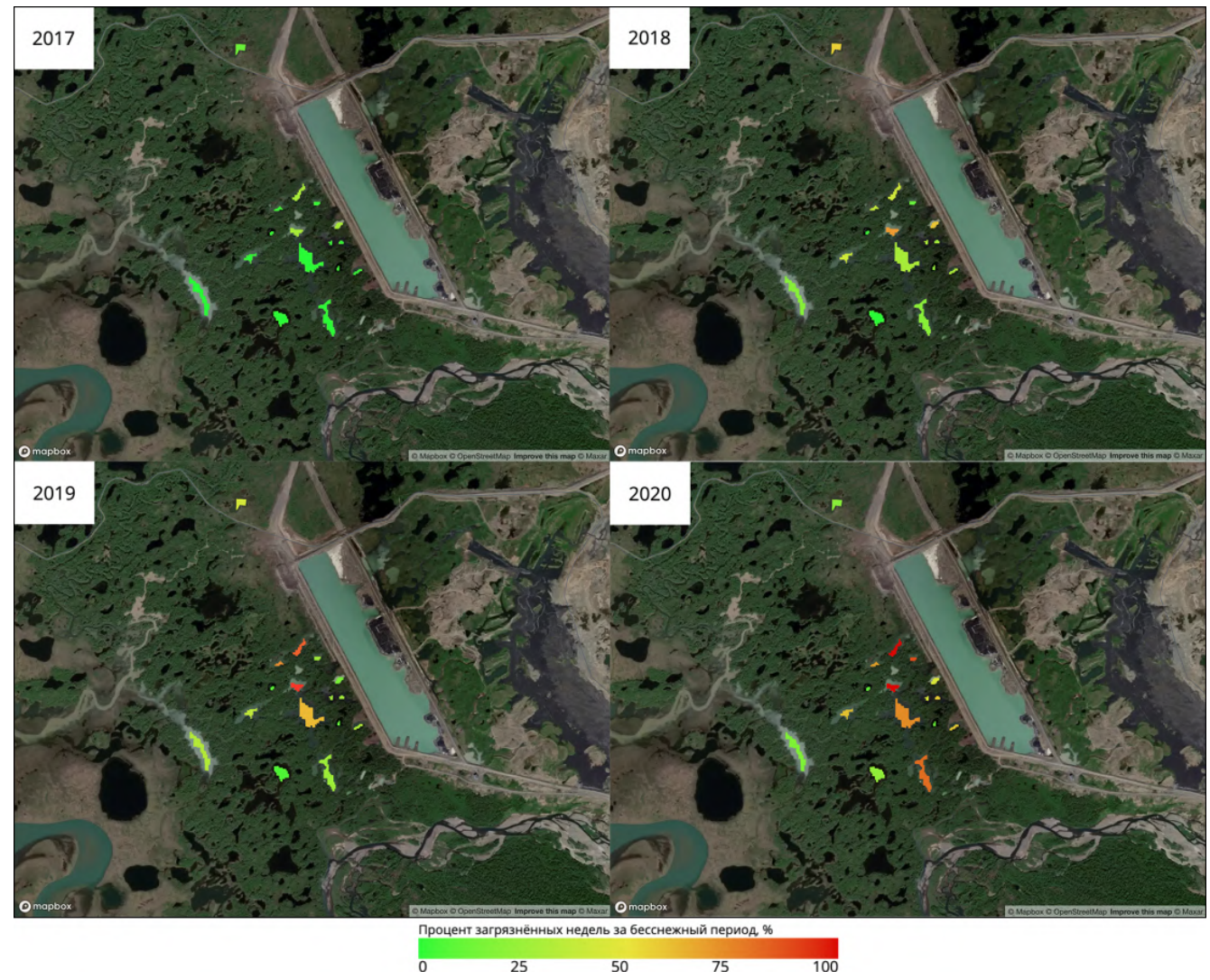

Figure 4: Pollution map of water bodies around the Novoe tailing dump (2017-2020). 
started to fill up in 2017. The data shown in Figure 4 reflect the increasing level of pollution of water bodies in the period from 2017 to 2020 , which indicates the likelihood that violations or mistakes could have been made in the design or construction of the tailing dump.

\section{Conclusions}

Thus, it can be noted that the new "object-oriented" approach to the use of remote sensing data allows solving quite diverse problems and organizing constant remote monitoring of various natural and natural-anthropogenic objects, investigating them in retrospect, and studying their current state. The subsystem is already being used in several projects, in particular for assessing the environmental impact of large industrial facilities. In this case, the set of monitored indicators can be quite easily expanded, and the subsystem can apply for research in various subject areas.

\section{Acknowledgments}

The study was supported by RFBR in framework of the scientific project No. 18-29-24121 MK using resources of the "IKI-Monitoring" Center for Collective Use [2].

\section{References}

[1] Konstantinova A.M., Balashov I.V., Kashnitskiy A.V., Loupian E.A., Proshin A.A., Senko K.S., Sychugov I.G. Organization of subsystems for working with observations of objects in services using GEOSMIS technology // Materials of the Eighteenth All-Russian Open Conference "Actual Problems of Remote Sensing of the Earth from Space". November 16-20, 2020. IKI RAN, 2020. P. 82. DOI:10.21046/18DZZconf-2020.

[2] Loupian E.A., Proshin A.A., Bourtsev M.A., Kashnitskiy A.V., Balashov I.V., Bartalev S.A., Konstantinova A.M., Kobets D.A., Mazurov A.A., Marchenkov V.V., Matveev A.M., Radchenko M.V., Sychugov I.G., Tolpin V.A., Uvarov I.A. Experience of development and operation of the IKI-Monitoring center for collective use of systems for archiving, processing and analyzing satellite data // Actual Problems of Remote Sensing of the Earth from Space. 2019. Vol. 16. No. 3. P. 151-170. DOI:10.21046/2070-7401-2019-16-3-151-170.

[3] Loupian E.A., Konstantinova A.M., Balashov I.V., Kashnitskiy A.V., Savorsky V.P., Panova O.Yu. Development of a system for analyzing the state of environment in areas of large industrial facilities, tailings and dumps // Actual Problems of Remote Sensing of the Earth from Space. 2020. Vol. 17. No. 7. P. 243-261. DOI:10.21046/2070-7401-2020-177-243-261.

[4] Loupian E.A., Proshin A.A., Burtsev M.A., Balashov I.V., Bartalev S.A., Efremov V.Yu., Kashnitskiy A.V., Mazurov A.A., Matveev A.M., Sudneva O.A., Sychugov I.G., Tolpin V.A., Uvarov I.A. IKI center for collective use of satellite data archiving, processing and analysis systems aimed at solving the problems of environmental study and monitoring // Sovremennye Problemy Distantsionnogo Zondirovaniya Zemli iz Kosmosa. 2015. Vol. 12. No. 5. P. 263-284. 